\title{
Voltage and Laser-Assisted Mode Atom Probe Tomography of Gallium Nitride
}

\author{
Nabil Dawahre, Gang Shen, Seongsin Margaret Kim, and Patrick Kung \\ Department of Electrical and Computer Engineering, The University of Alabama, Tuscaloosa, USA
}

Because of their unique physical properties, many of which arise from a direct bandgap ranging from 6.2 to $0.7 \mathrm{eV}$, Gallium Nitride $(\mathrm{GaN})$ based semiconductors are leading optoelectronic materials that have enabled blue and green lasers, ultraviolet-blue-green-amber and white light emitting diodes, as well as the next generation RF-microwave devices, power amplifiers and high temperature electronics [1]. Atom probe tomography (APT) has recently been receiving increasing attention as a characterization technique for semiconductors which can bridge the gap between high-resolution sub-nanometer imaging and high sensitivity chemical element identification, although much remains to be understood in the APT of GaN. Following developments in atom-probe instrumentation during the past few years, preference has widely been given to using laser-assisted APT in the case of semiconductors as they are much less conductive than metals [2]. Here, we compare the voltage-pulsed and laser-assisted APT of GaN.

Specimens were prepared using a dual-beam focused ion beam from several (0001) oriented undoped $\mathrm{GaN}$ thin films grown by metalorganic vapor phase epitaxy that are typically used in high electron mobility transistors. The specimen tip was imaged on a Transmission Electron Microscope (TEM), which revealed a $60 \mathrm{~nm}$ in radius and a 4 degree half shank angle (Figure 1 (a)). The samples were analyzed using a Local Electrode Atom Probe (LEAP) 3000X Si under ultra-high vacuum $\left(\sim 10^{-11}\right.$ Torr) and at cryogenic temperatures $(\sim 60 \mathrm{~K})$.

During voltage mode APT, the detector events histogram (Figure 1 (b)) usually shows a 6 foldsymmetry consistent with the c-axis of GaN being oriented along the axis of the specimen tip. Laserassisted APT of the same tips did not reveal as clear a symmetry pattern. The mass-to-charge $(\mathrm{m} / \mathrm{c})$ spectrum for the voltage mode is shown in Figure 2(a). Although APT was achievable for GaN using voltage pulsing, a larger number of peaks were detected, primarily located between $\mathrm{m} / \mathrm{c} 80$ and 95 , which makes identification more challenging and is an indication of a more complex field evaporation mechanism in this mode, especially in the case of undoped GaN. Because this material is much less conductive than other narrower bandgap semiconductors, one expects a significant voltage drop between the base of the sample and the tip apex. This can be more readily observed in the tailing on the high $\mathrm{m} / \mathrm{c}$ side of the peaks, such as for the $\mathrm{Ga}^{+}$shown in Figure 2(c). The detection of ions in the tail can be interpreted as these ions exhibiting a longer time-of-flight to reach the detector than those contributing to the peak, which is due to the voltage drop within the semiconductor material.

By contrast, the laser mode yielded cleaner spectra with fewer and narrower peaks that were easily identified (Figure 2(b)). This is primarily due to a quite different evaporation process in laser-assisted APT, which is widely believed to involve local heating at/near the tip instead of electric field-only induced evaporation [3]. In our experiments, a $532 \mathrm{~nm}$ laser was used with a $<\sim 15$ ps pulse duration. The potential physical mechanisms at the origin of local heating in wide bandgap GaN, which is transparent to a 532 laser beam, include thermalization after free carrier absorption, as well as absorption by defects, and two-photon absorption [4] which may not be negligible since the carrier concentration in these semiconductors decreases dramatically at low temperature. The influence of the pulsed laser analysis was performed by tuning the laser pulse energy from 2 to $0.14 \mathrm{~nJ}$. Figure 3 shows the total concentrations of $\mathrm{Ga}$ and $\mathrm{N}$ detected in the mass spectra as a function of pulse energy. Although stoichiometric values of Ga: $\mathrm{N}$ are achievable at the lowest pulse energy, as the it is increased, the 
proportion of $\mathrm{N}$ quickly drops to under $20 \%$. This is most likely due to the formation of neutral $\mathrm{N}_{2}$ molecules that are not detected in the mass spectrometer. Obviously, this phenomenon is facilitated at high pulse energies, i.e. larger heat.

\section{References:}

[1] F. Ren and J. Zolper. "Wide Energy Bandgap Electronic Devices" (World Scientific Pub Co., River Edge, NJ, 2004)

[2] A. Shariq, S Mutas, K. Wedderhoff et al. Ultramicroscopy, 109 (2009) 472

[3] A. Cerezo, C.R.M. Grovenor, and G.D.W. Smith. Applied Physics Letters, 88 (2006) 154103

[4] A.M. Streltsov, K.D. Moll, A.L. Gaeta et al. Applied Physics Letters, 75 (1999) 3778

[5] The authors acknowledge funding from the National Science Foundation (DMR-0907558) and the Defense Threat Reduction Agency (HDTRA1-11-1-0071).
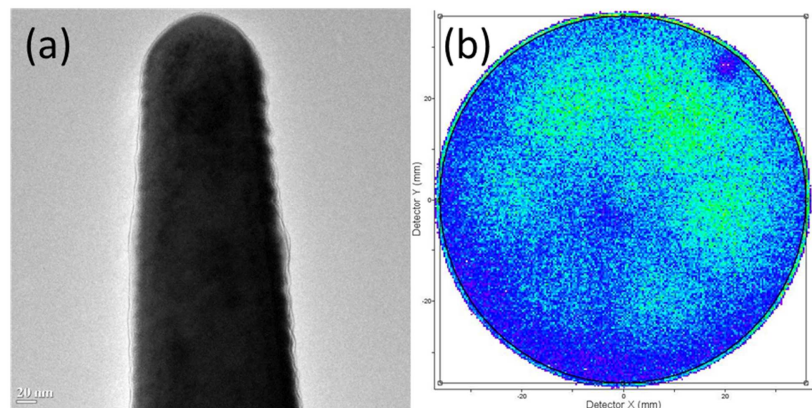

Figure 1. (a) High Resolution TEM image of a typical specimen tip for APT. (b) Histogram of the detector events for $\mathrm{GaN}$ observed under voltage mode APT.
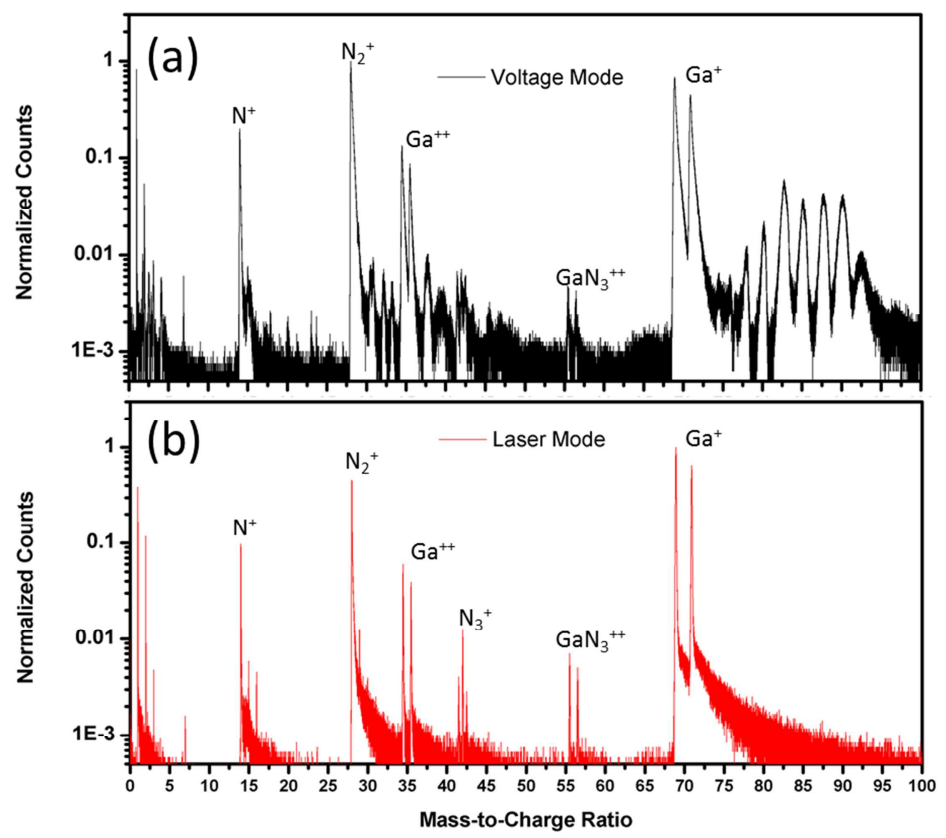

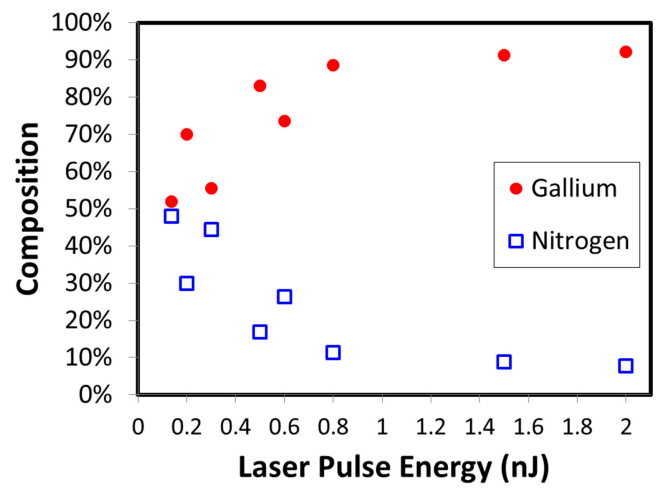

Figure 3. Concentrations of $\mathrm{Ga}$ and $\mathrm{N}$ atoms detected as a function of laser pulse energy.

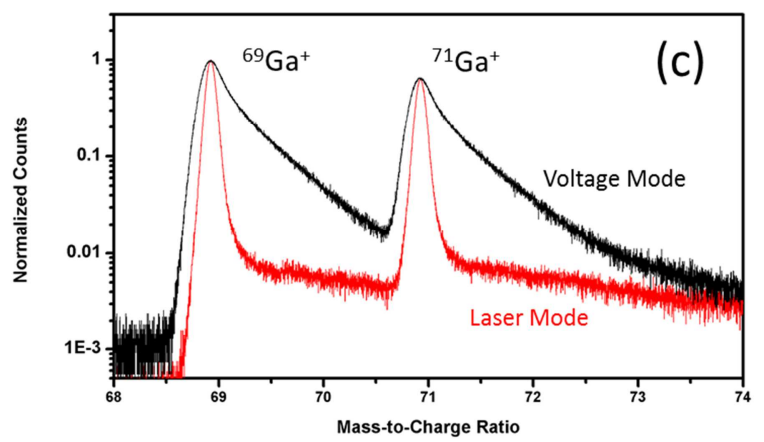

Figure 2. APT mass-to-charge spectra of GaN: (a) under voltage mode, (b) laser-assisted mode. (c) Mass spectra of voltage and laser modes for $\mathrm{Ga}^{+}$peak. 